\title{
Stability and Bifurcation in a Delayed Holling-Tanner Predator-Prey System with Ratio-Dependent Functional Response
}

\author{
Juan Liu, ${ }^{1}$ Zizhen Zhang, ${ }^{2}$ and Ming Fu' ${ }^{2}$ \\ ${ }^{1}$ Department of Science, Bengbu College, Bengbu, Anhui 233030, China \\ ${ }^{2}$ School of Management Science and Engineering, Anhui University of Finance and Economics, \\ Bengbu, Anhui 233030, China
}

Correspondence should be addressed to Juan Liu, liujuan7216@163.com

Received 9 November 2012; Revised 21 November 2012; Accepted 21 November 2012

Academic Editor: C. Conca

Copyright (C) 2012 Juan Liu et al. This is an open access article distributed under the Creative Commons Attribution License, which permits unrestricted use, distribution, and reproduction in any medium, provided the original work is properly cited.

\begin{abstract}
We analyze a delayed Holling-Tanner predator-prey system with ratio-dependent functional response. The local asymptotic stability and the existence of the Hopf bifurcation are investigated. Direction of the Hopf bifurcation and the stability of the bifurcating periodic solutions are studied by deriving the equation describing the flow on the center manifold. Finally, numerical simulations are presented for the support of our analytical findings.
\end{abstract}

\section{Introduction}

Predator-prey dynamics has long been and will continue to be of interest to both applied mathematicians and ecologists due to its universal existence and importance [1]. Although the early Lotka-Volterra model has given way to more sophisticated models from both a mathematical and biological point of view, it has been challenged by ecologists for its functional response suffers from paradox of enrichment and biological control paradox. The ratio-dependent models are discussed as a solution to these difficulties and found to be a more reasonable choice for many predator-prey interactions [2-4]. One type of the ratio-dependent models which plays a special role in view of the interesting dynamics it possesses is the ratio-dependent Holling-Tanner predator-prey system [5, 6]. A ratiodependent Holling-Tanner predator-prey system takes the form of

$$
\begin{gathered}
\frac{d N}{d t}=r N(t)\left(1-\frac{N(t)}{k}\right)-\frac{m N(t) P(t)}{N(t)+q P(t)}, \\
\frac{d P}{d t}=P(t)\left[s\left(1-h \frac{P(t)}{N(t)}\right)\right],
\end{gathered}
$$


where $N(t)$ and $P(t)$ represent the population of prey species and predator species at time $t$. It is assumed that in the absence of the predator, the prey grows logistically with carrying $k$ and intrinsic growth rate $r$. The predator growth equation is of logistic type with a modification of the conventional one. The parameter $m$ represents the maximal predator per capita consumption rate, and $q$ is the half capturing saturation constant. The parameter $s$ is the intrinsic growth rate of the predator and $h$ is the number of prey required to support one predator at equilibrium, when $y$ equals $x / h$. All the parameters are assumed to be positive.

Liang and Pan [6] established the sufficient conditions for the global stability of positive equilibrium of system (1.1) by constructing Lyapunov function. Considering the effect of time delays on the system, Saha and Chakrabarti [7] considered the following delayed system

$$
\begin{gathered}
\frac{d N}{d t}=r N(t)\left(1-\frac{N(t-\tau)}{k}\right)-\frac{m N(t) P(t)}{N(t)+q P(t)} \\
\frac{d P}{d t}=P(t)\left[s\left(1-h \frac{P(t)}{N(t)}\right)\right]
\end{gathered}
$$

where $\tau$ is the negative feedback delay of the prey. Saha and Chakrabarti [7] proved that the system (1.2) is permanent under certain conditions and obtained the conditions for the local and global stability of the positive equilibrium. It is well known that studies on dynamical systems not only involve a discussion of stability and persistence, but also involve many dynamical behaviors such as periodic phenomenon, bifurcation, and chaos [8-10]. In particular, the Hopf bifurcation has been studied by many authors [11-13]. Based on this consideration and since both species are growing logistically, we consider the Hopf bifurcation of the following system with two delays:

$$
\begin{gathered}
\frac{d N}{d t}=r N(t)\left(1-\frac{N\left(t-\tau_{1}\right)}{k}\right)-\frac{m N(t) P(t)}{N(t)+q P(t)}, \\
\frac{d P}{d t}=P(t)\left[s\left(1-h \frac{P\left(t-\tau_{2}\right)}{N\left(t-\tau_{2}\right)}\right)\right]
\end{gathered}
$$

where $\tau_{1}$ and $\tau_{2}$ represent the negative feedbacks in prey and predator growth.

Before proceeding further we nondimensionalize our model system (1.3) with the following scaling $r t \rightarrow t, r \tau_{1} \rightarrow \tau_{1}, r \tau_{2} \rightarrow \tau_{2}, N(t) / k \rightarrow x(t), m P(t) / r k \rightarrow y(t)$. Then we get the nondimensional form of system (1.3):

$$
\begin{gathered}
\frac{d x}{d t}=x(t)\left(1-x\left(t-\tau_{1}\right)\right)-\frac{x(t) y(t)}{x(t)+\alpha y(t)} \\
\frac{d y}{d t}=\beta y(t)\left(\delta-\frac{y\left(t-\tau_{2}\right)}{x\left(t-\tau_{2}\right)}\right)
\end{gathered}
$$

where $\alpha=q r / m, \beta=s h / m, \delta=m / h r$.

This paper is organized as follows. In the next section, we will consider the local stability of the positive equilibrium and the existence of Hopf bifurcation of system (1.4). In Section 3, we can determine the direction of the Hopf bifurcations and the stability of 
the bifurcating periodic solutions. Some numerical simulations are also given to illustrate the theoretical prediction in Section 4.

\section{Local Stability and Hopf Bifurcation}

Considering the ecological significance of system (1.4), we are interested only in the positive equilibrium of system (1.4). It is not difficult to verify that system (1.4) has a unique positive equilibrium $E\left(x_{0}, y_{0}\right)$, where $x_{0}=(1+\alpha \delta-\delta) /(1+\alpha \delta), y_{0}=\delta x_{0}$ if $\left(H_{1}\right): 1+\alpha \delta>\delta$ holds.

Let $x(t)=u_{1}(t)+x_{0}, y(t)=u_{2}(t)+y_{0}$, and we still denote $u_{1}(t)$ and $u_{2}(t)$ by $x(t)$ and $y(t)$, respectively, then system (1.4) can be rewritten as

$$
\begin{aligned}
& \frac{d x(t)}{d t}=a_{11} x(t)+a_{12} y(t)+b_{11} x\left(t-\tau_{1}\right)+\sum_{i+j+k \geq 2} f_{1}^{i j k} x^{i} y^{j} x^{k}\left(t-\tau_{1}\right), \\
& \frac{d y(t)}{d t}=b_{21} x\left(t-\tau_{2}\right)+b_{22} y\left(t-\tau_{2}\right)+\sum_{i+j+k \geq 2} f_{2}^{i j k} y^{i} x^{j}\left(t-\tau_{2}\right) y^{k}\left(t-\tau_{2}\right),
\end{aligned}
$$

where

$$
\begin{gathered}
a_{11}=1-x_{0}-\frac{\alpha y_{0}^{2}}{\left(x_{0}+\alpha y_{0}\right)^{2}}, \quad a_{12}=-\frac{x_{0}^{2}}{\left(x_{0}+\alpha y_{0}\right)^{2}}, \\
b_{11}=-x_{0}, \quad b_{21}=\frac{\beta y_{0}^{2}}{x_{0}^{2}}, \quad b_{22}=-\frac{\beta y_{0}}{x_{0}}, \\
f_{1}^{i j k}=\left.\frac{1}{i ! j ! k !} \frac{\partial^{i+j+k} f_{1}}{\partial x^{i} \partial y^{j} \partial x^{k}\left(t-\tau_{1}\right)}\right|_{\left(x_{0}, y_{0}\right)}, \quad f_{2}^{i j k}=\left.\frac{1}{i ! j ! k !} \frac{\partial^{i+j+k} f_{2}}{\partial y^{i} \partial x^{j}\left(t-\tau_{2}\right) \partial y^{k}\left(t-\tau_{2}\right)}\right|_{\left(x_{0}, y_{0}\right)}, \\
f_{1}=x(t)\left(1-x\left(t-\tau_{1}\right)\right)-\frac{x(t) y(t)}{x(t)+\alpha y(t)}, \quad f_{2}=\beta y(t)\left(\delta-\frac{y\left(t-\tau_{2}\right)}{x\left(t-\tau_{2}\right)}\right) .
\end{gathered}
$$

Then the linearized system of (2.1) is

$$
\begin{gathered}
\frac{d x(t)}{d t}=a_{11} x(t)+a_{12} y(t)+b_{11} x\left(t-\tau_{1}\right), \\
\frac{d y(t)}{d t}=b_{21} x\left(t-\tau_{2}\right)+b_{22} y\left(t-\tau_{2}\right) .
\end{gathered}
$$

The characteristic equation of (2.3) is

$$
\lambda^{2}+A \lambda+B \lambda e^{-\lambda \tau_{1}}+(C \lambda+D) e^{-\lambda \tau_{2}}+E e^{-\lambda\left(\tau_{1}+\tau_{2}\right)}=0,
$$

where

$$
A=-a_{11}, \quad B=-b_{11}, \quad C=-b_{22}, \quad D=a_{11} b_{22}-a_{12} b_{21}, \quad E=b_{11} b_{22} .
$$


Case $1\left(\tau_{1}=\tau_{2}=0\right)$. Equation (2.4) reduces to

$$
\lambda^{2}+(A+B+C) \lambda+D+E=0
$$

If the condition $\left(\mathrm{H}_{2}\right): A+B+C>0$ and $D+E>0$ holds, it is clear that roots of (2.6) must have negative real parts.

Case $2\left(\tau_{1}>0, \tau_{2}=0\right)$. Equation (2.4) becomes

$$
\lambda^{2}+(A+C) \lambda+D+(B \lambda+E) e^{-\lambda \tau_{1}}=0 .
$$

Let $\lambda=i \omega_{1}\left(\omega_{1}>0\right)$ be a root of (2.7). Then, we have

$$
\begin{gathered}
B \omega_{1} \sin \tau_{1} \omega_{1}+E \cos \tau_{1} \omega_{1}=\omega_{1}^{2}-D \\
B \omega_{1} \cos \tau_{1} \omega_{1}-E \sin \tau_{1} \omega_{1}=-(A+C) \omega_{1} .
\end{gathered}
$$

From (2.8), we can get

$$
\omega_{1}^{4}+\left((A+C)^{2}-B^{2}-2 D\right) \omega_{1}^{2}+D^{2}-E^{2} .
$$

If the condition $\left(H_{3}\right): D^{2}-E^{2}<0$ holds, then (2.9) has a unique positive root $\omega_{10}$

$$
\omega_{10}=\sqrt{\frac{-\left((A+C)^{2}-B^{2}-2 D\right)+\sqrt{\left((A+C)^{2}-B^{2}-2 D\right)^{2}-4\left(D^{2}-E^{2}\right)}}{2}} .
$$

The corresponding critical value of time delay $\tau_{10}$ is

$$
\tau_{10}=\frac{1}{\omega_{10}} \arccos \frac{(E-A B-B C) \omega_{10}^{2}-D E}{B^{2} \omega_{10}^{2}+E^{2}}+\frac{2 n \pi}{\omega_{10}}, \quad n=0,1,2, \ldots
$$

Next, differentiating (2.7) with respect to $\tau_{1}$ and substituting $\tau_{1}=\tau_{10}$, then we get

$$
\operatorname{Re}\left\{\frac{d \lambda}{d \tau_{1}}\right\}_{\tau_{1}=\tau_{10}}^{-1}=\frac{2 \omega_{10}^{2}+(A+C)^{2}-B^{2}-2 D}{(A+C)^{2} \omega_{10}^{2}+\left(\omega_{10}^{2}-D\right)^{2}}
$$

From (2.10) and (2.12), we have

$$
\operatorname{Re}\left\{\frac{d \lambda}{d \tau_{1}}\right\}_{\tau_{1}=\tau_{10}}^{-1}=\frac{\sqrt{\left((A+C)^{2}-B^{2}-2 D\right)^{2}-4\left(D^{2}-E^{2}\right)}}{(A+C)^{2} \omega_{10}^{2}+\left(\omega_{10}^{2}-D\right)^{2}}
$$


Therefore, if the condition $\left(H_{3}\right): D^{2}-E^{2}<0$ holds, then $\operatorname{Re}\left\{d \lambda / d \tau_{1}\right\}_{\tau_{1}=\tau_{10}}^{-1}>0$. Thus, we have the following results.

Theorem 2.1. For system (1.4), if the conditions $\left(H_{2}\right)-\left(H_{3}\right)$ hold, then the positive equilibrium $E\left(x_{0}, y_{0}\right)$ of system $(1.4)$ is asymptotically stable for $\tau \in\left[0, \tau_{10}\right)$ and unstable when $\tau>\tau_{10}$, system (1.4) undergoes a Hopf bifurcation at $E\left(x_{0}, y_{0}\right)$ when $\tau_{1}=\tau_{10}$.

Case $3\left(\tau_{1}=0, \tau_{2}>0\right)$. Equation (2.4) becomes

$$
\lambda^{2}+(A+B) \lambda+(C \lambda+D+E) e^{-\lambda \tau_{2}}=0 .
$$

Let $\lambda=i \omega_{2}\left(\omega_{2}>0\right)$ be a root of (2.14). Then, we get

$$
\begin{gathered}
C \omega_{2} \sin \tau_{2} \omega_{2}+(D+E) \cos \tau_{2} \omega_{2}=\omega_{2}^{2} \\
C \omega_{2} \cos \tau_{2} \omega_{2}-(D+E) \sin \tau_{2} \omega_{2}=-(A+B) \omega_{2} .
\end{gathered}
$$

It follows that

$$
\omega_{2}^{4}+\left((A+B)^{2}-C^{2}\right) \omega_{2}^{2}-(D+E)^{2} .
$$

If the condition $\left(H_{2}\right)$ holds, then $-(D+E)^{2}<0$. Thus, $(2.16)$ has a unique positive root $\omega_{20}$,

$$
\omega_{20}=\sqrt{\frac{-\left((A+B)^{2}-C^{2}\right)+\sqrt{\left((A+B)^{2}-C^{2}\right)^{2}+4(D+E)^{2}}}{2}} .
$$

The corresponding critical value of time delay $\tau_{20}$ is

$$
\tau_{20}=\frac{1}{\omega_{20}} \arccos \frac{(D+E-A C-B C) \omega_{10}^{2}}{C^{2} \omega_{20}^{2}+(D+E)^{2}}+\frac{2 n \pi}{\omega_{20}}, \quad n=0,1,2, \ldots
$$

Similar as in Case 2, we know that if the condition $\left(\mathrm{H}_{2}\right)$ holds, then we have

$$
\operatorname{Re}\left\{\frac{d \lambda}{d \tau_{2}}\right\}_{\tau_{2}=\tau_{20}}^{-1}=\frac{\sqrt{\left((A+B)^{2}-C^{2}\right)^{2}+4(D+E)^{2}}}{\omega_{20}^{4}+(A+B)^{2} \omega_{20}^{2}}>0
$$

In conclusion, we have the following results.

Theorem 2.2. For system (1.4), if the condition $\left(H_{2}\right)$ holds, then the positive equilibrium $E\left(x_{0}, y_{0}\right)$ of system (1.4) is asymptotically stable for $\tau \in\left[0, \tau_{20}\right)$ and unstable when $\tau>\tau_{20}$, system (1.4) undergoes a Hopf bifurcation at $E\left(x_{0}, y_{0}\right)$ when $\tau_{1}=\tau_{20}$. 
Case $4\left(\tau_{1}=\tau_{2}=\tau>0\right)$. Equation (2.4) becomes

$$
\lambda^{2}+A \lambda+((B+C) \lambda+D) e^{-\lambda \tau}+E e^{-2 \lambda \tau}=0
$$

Multiplying $e^{\lambda \tau}$ on both sides of (2.20), we have

$$
(B+C) \lambda+D+\left(\lambda^{2}+A \lambda\right) e^{\lambda \tau}+E e^{-\lambda \tau}=0 .
$$

Let $\lambda=i \omega(\omega>0)$ be a root of (2.21). Then, we get

$$
\begin{gathered}
A \omega \sin \tau \omega-\left(E-\omega^{2}\right) \cos \tau \omega=D \\
A \omega \cos \tau \omega-\left(E+\omega^{2}\right) \sin \tau \omega=-(B+C) \omega .
\end{gathered}
$$

Then, we can get

$$
\sin \tau \omega=\frac{m_{3} \omega^{3}+m_{1} \omega}{\omega^{4}+n_{2} \omega^{2}+n_{0}}, \quad \cos \tau \omega=\frac{m_{2} \omega^{2}+m_{0}}{\omega^{4}+n_{2} \omega^{2}+n_{0}},
$$

where

$$
\begin{gathered}
m_{0}=D E, \quad m_{1}=A D-E(B+C), \quad m_{2}=D-A(B+C), \\
m_{3}=B+C, \quad n_{0}=-E^{2}, \quad n_{2}=A^{2} .
\end{gathered}
$$

Thus, we can obtain

$$
\omega^{8}+A_{3} \omega^{6}+A_{2} \omega^{4}+A_{1} \omega^{2}+A_{0}=0,
$$

with

$$
\begin{aligned}
& A_{0}=n_{0}^{2}-m_{0}^{2}, \quad A_{1}=2 n_{0} n_{2}-m_{1}^{2}-2 m_{0} m_{2}, \\
& A_{2}=2 n_{0}-2 m_{1} m_{3}-m_{2}^{2}, \quad A_{3}=2 n_{2}-m_{3}^{2} .
\end{aligned}
$$

Let $e=\omega^{2}$, then (2.25) can be transformed into the following form

$$
e^{4}+A_{3} e^{3}+A_{2} e^{2}+A_{1} e+A_{0}=0
$$

Next, we suppose that $\left(H_{4}\right)$ : (2.27) has at least one positive root. Without loss of generality, we suppose that it has four positive roots which are denoted as $e_{1}, e_{2}, e_{3}$, and $e_{4}$. Thus, (2.25) 
has four positive roots $\omega_{k}=\sqrt{e_{k}}, k=1,2,3,4$. The corresponding critical value of time delay $\tau_{k}^{(j)}$ is

$$
\tau_{k}^{(j)}=\frac{1}{\omega_{k}} \arccos \frac{m_{2} \omega^{2}+m_{0}}{\omega^{4}+n_{2} \omega^{2}+n_{0}}+\frac{2 j \pi}{\omega_{k}}, \quad k=1,2,3,4, j=0,1,2 \ldots
$$

Let $\tau_{0}=\min \left\{\tau_{k}^{(0)}\right\}, k \in\{1,2,3,4\}, \omega_{0}=\omega_{k_{0}}$.

Differentiating (2.21) regarding $\tau$ and substituting $\tau=\tau_{0}$, we obtain

$$
\operatorname{Re}\left\{\frac{d \lambda}{d \tau}\right\}_{\tau=\tau_{0}}^{-1}=\frac{M_{R} N_{R}+M_{I} N_{I}}{N_{R}^{2}+N_{I}^{2}}
$$

where

$$
\begin{gathered}
M_{R}=B+C+A \cos \tau_{0} \omega_{0}-2 \omega_{0} \sin \tau_{0} \omega_{0}, \quad M_{I}=A \sin \tau_{0} \omega_{0}+2 \omega_{0} \cos \tau_{0} \omega_{0} \\
N_{R}=\left(A \omega_{0}^{2}+E\right) \cos \tau_{0} \omega_{0}-\omega_{0}^{3} \sin \tau_{0} \omega_{0}, \quad N_{I}=\left(A \omega_{0}^{2}-E\right) \sin \tau_{0} \omega_{0}+\omega_{0}^{3} \cos \tau_{0} \omega_{0} .
\end{gathered}
$$

Obviously, if the condition $\left(H_{5}\right): M_{R} N_{R}+M_{I} N_{I} \neq 0$ holds, then $\operatorname{Re}\{d \lambda / d \tau\}_{\tau=\tau_{0}}^{-1} \neq 0$. Namely, the transversality condition is satisfied if $H_{5}$ holds. From the above analysis, we have the following theorem.

Theorem 2.3. For system (1.4), if the conditions $\left(H_{2}\right),\left(H_{4}\right)$, and $\left(H_{5}\right)$ hold, then the positive equilibrium $E\left(x_{0}, y_{0}\right)$ of system (1.4) is asymptotically stable for $\tau \in\left[0, \tau_{0}\right)$ and unstable when $\tau>\tau_{0}$, system (1.4) undergoes a Hopf bifurcation at $E\left(x_{0}, y_{0}\right)$ when $\tau=\tau_{0}$.

Case $5\left(\tau_{1} \neq \tau_{2}\right.$ and $\left.\tau_{1}>0, \tau_{2}>0\right)$. We consider (2.4) with $\tau_{2}$ in its stable interval and $\tau_{1}$ is considered as a parameter. Let $\lambda=i \omega_{1}^{\prime}\left(\omega_{1}^{\prime}>0\right)$ be the root of (2.4). Then we have

$$
\begin{aligned}
& M \sin \tau_{1} \omega_{1}^{\prime}+N \cos \tau_{1} \omega_{1}^{\prime}=P, \\
& M \cos \tau_{1} \omega_{1}^{\prime}-N \sin \tau_{1} \omega_{1}^{\prime}=Q,
\end{aligned}
$$

where

$$
\begin{gathered}
M=B \omega_{1}^{\prime}-E \sin \tau_{2} \omega_{1}^{\prime}, \quad N=E \cos \tau_{2} \omega_{1}^{\prime}, \\
P=\left(\omega_{1}^{\prime}\right)^{2}-C \omega_{1}^{\prime} \sin \tau_{2} \omega_{1}^{\prime}-D \cos \tau_{2} \omega_{1}^{\prime}, \\
Q=-A \omega_{1}^{\prime}-C \omega_{1}^{\prime} \cos \tau_{2} \omega_{1}^{\prime}+D \sin \tau_{2} \omega_{1}^{\prime} .
\end{gathered}
$$

It follows that

$$
f_{1}\left(\omega_{1}^{\prime}\right)+f_{2}\left(\omega_{1}^{\prime}\right) \cos \tau_{2} \omega_{1}^{\prime}+f_{3}\left(\omega_{1}^{\prime}\right) \sin \tau_{2} \omega_{1}^{\prime}=0,
$$


where

$$
\begin{gathered}
f_{1}\left(\omega_{1}^{\prime}\right)=\left(\omega_{1}^{\prime}\right)^{4}+\left(A^{2}-B^{2}+C^{2}\right)\left(\omega_{1}^{\prime}\right)^{2}+D^{2}-E^{2} \\
f_{2}\left(\omega_{1}^{\prime}\right)=2(A C-D)\left(\omega_{1}^{\prime}\right)^{2}, \quad f_{3}\left(\omega_{1}^{\prime}\right)=-2 C\left(\omega_{1}^{\prime}\right)^{3}+(2 B E-2 A D) \omega_{1}^{\prime} .
\end{gathered}
$$

Suppose that $\left(H_{6}\right):(2.33)$ has at least finite positive roots.

If the condition $\left(H_{6}\right)$ holds, we denote the roots of $(2.33)$ as $\omega_{11}^{\prime}, \omega_{12}^{\prime}, \ldots \omega_{1 k}^{\prime}$. Then, for every fixed $\omega_{1 i}^{\prime}(i=1,2, \ldots k)$, the corresponding critical value of time delay $\left\{\tau_{1 i}^{(j)} \mid j=\right.$ $0,1,2, \ldots\}$ is

$$
\tau_{1 i}^{(j)}=\frac{1}{\omega_{i}} \arccos \frac{M Q+P N}{M^{2}+N^{2}}+\frac{2 j \pi}{\omega_{i}}, \quad i=1,2, \ldots k, j=0,1,2, \ldots
$$

Let $\tau_{1 *}=\min \left\{\tau_{1 i}^{(j)} \mid i=1,2, \ldots, k, j=0,1,2, \ldots\right\}$. The corresponding purely imaginary roots of (2.33) are denoted as $\pm i \omega_{*}$. Next, we give the following assumption. $\left(H_{7}\right)$ : $\left\{d \operatorname{Re}(\lambda) / d \tau_{1}\right\}_{\tau_{1}=\tau_{1 *}}^{-1} \neq 0$. Hence, we have the following theorem.

Theorem 2.4. Suppose that the conditions $\left(H_{2}\right),\left(H_{6}\right)$, and $\left(H_{7}\right)$ hold and $\tau_{2} \in\left(0, \tau_{20}\right)$. The positive equilibrium $E\left(x_{0}, y_{0}\right)$ of system (1.4) is asymptotically stable for $\tau_{1} \in\left[0, \tau_{1 *}\right)$ and unstable when $\tau_{1}>\tau_{1 *}$, system (1.4) undergoes a Hopf bifurcation at $E\left(x_{0}, y_{0}\right)$ when $\tau_{1}=\tau_{0}$.

\section{Direction and Stability of Bifurcated Periodic Solutions}

In this section, we will employ the normal form method and center manifold theorem introduced by Hassard [14] to determine the direction of Hopf bifurcation and stability of bifurcating periodic solutions of system (1.4) at $\tau_{1}=\tau_{2}=\tau=\tau_{0}$.

We denote $\tau$ as $\tau=\tau_{0}+\mu, \mu \in R, t=s \tau$, Then $\mu=0$ is the Hopf bifurcation value of system (1.4). For convenience, we first rescale the time by $s \rightarrow t / \tau, x(s \tau)=x_{1}(s), y(s \tau)=$ $x_{2}(s)$ and still denote $s=t$, then system (1.4) can be transformed to the following form:

$$
\dot{u}(t)=L_{\mu} u_{t}+F\left(\mu, u_{t}\right)
$$

where

$$
\begin{gathered}
u(t)=(x(t), y(t))^{T} \in C\left([-1,0], R^{2}\right), \\
u_{t}(\theta)=u(t+\theta)=(x(t+\theta), y(t+\theta))^{T} \in C\left([-1,0], R^{2}\right)
\end{gathered}
$$

and $L_{\mu}, F$ are given by

$$
\begin{gathered}
L_{\mu} \phi=\left(\tau_{0}+\mu\right)\left(\begin{array}{cc}
a_{11} & a_{12} \\
0 & 0
\end{array}\right)\left(\begin{array}{l}
\phi_{1}(0) \\
\phi_{2}(0)
\end{array}\right)+\left(\tau_{0}+\mu\right)\left(\begin{array}{ll}
b_{11} & 0 \\
b_{21} & b_{22}
\end{array}\right)\left(\begin{array}{l}
\phi_{1}(-1) \\
\phi_{2}(-1)
\end{array}\right), \\
F(\mu, \phi)=\left(\tau_{0}+\mu\right)\left(F_{1}, F_{2}\right)^{T},
\end{gathered}
$$


where $\phi(\theta)=\left(\phi_{1}(\theta), \phi_{2}(\theta)\right)^{T} \in C\left([-1,0], R^{2}\right)$, and

$$
\begin{aligned}
F_{1}= & a_{13} \phi_{1}^{2}(0)-\phi_{1}(0) \phi_{1}(-1)+a_{14} \phi_{1}(0) \phi_{2}(0)+a_{15} \phi_{2}^{2}(0)+a_{16} \phi_{1}^{3}(0) \\
& +a_{17} \phi_{1}^{2}(0) \phi_{2}(0)+a_{18} \phi_{1}(0) \phi_{2}^{2}(0)+a_{19} \phi_{2}^{3}(0)+\cdots, \\
F_{2}= & b_{23} \phi_{1}^{2}(-1)+b_{24} \phi_{1}(-1) \phi_{2}(0)+b_{25} \phi_{1}(-1) \phi_{2}(-1)+b_{26} \phi_{2}(0) \phi_{2}(-1) \\
& +b_{27} \phi_{1}^{3}(-1) \phi_{3}(-1)+b_{28} \phi_{1}^{2}(-1) \phi_{2}(0)+b_{29} \phi_{1}^{2}(-1) \phi_{2}(-1)+\cdots,
\end{aligned}
$$

with

$$
\begin{gathered}
a_{13}=\frac{\alpha y_{0}^{2}}{\left(x_{0}+\alpha y_{0}\right)^{3}}, \quad a_{14}=-\frac{2 \alpha x_{0} y_{0}}{\left(x_{0}+\alpha y_{0}\right)^{3}}, \quad a_{15}=\frac{\alpha x_{0}^{2}}{\left(x_{0}+\alpha y_{0}\right)^{3}}, \\
a_{16}=-\frac{\alpha y_{0}^{2}}{\left(x_{0}+\alpha y_{0}\right)^{4}}, \quad a_{17}=\frac{2 \alpha x_{0} y_{0}-\alpha^{2} y_{0}^{2}}{\left(x_{0}+\alpha y_{0}\right)^{4}}, \quad a_{18}=\frac{2 \alpha^{2} x_{0} y_{0}-\alpha x_{0}^{2}}{\left(x_{0}+\alpha y_{0}\right)^{4}}, \\
a_{19}=-\frac{\alpha^{2} y_{0}^{2}}{\left(x_{0}+\alpha y_{0}\right)^{4}}, \quad b_{23}=-\frac{\beta \delta^{2}}{x_{0}}, \quad b_{24}=\frac{\beta \delta}{x_{0}}, \quad b_{25}=\frac{\beta \delta}{x_{0}}, \\
b_{26}=-\frac{\beta}{x_{0}}, \quad b_{27}=\frac{\beta \delta^{2}}{x_{0}^{2}}, \quad b_{28}=-\frac{\beta \delta}{x_{0}^{2}}, \quad b_{29}=-\frac{\beta \delta}{x_{0}^{2}} .
\end{gathered}
$$

Hence, by the Riesz representation theorem, there exists a $2 \times 2$ matrix function $\eta(\theta, \mu)$ : $[-1,0] \rightarrow R^{2}$ whose elements are of bounded variation such that

$$
L_{\mu} \phi=\int_{-1}^{0}[d \eta(\theta, \mu)] \phi(\theta), \quad \phi \in C\left([-1,0], R_{+}^{3}\right) .
$$

In fact, we choose

$$
\eta(\theta, \mu)=\left(\tau_{0}+\mu\right)\left(\begin{array}{cc}
a_{11} & a_{12} \\
0 & 0
\end{array}\right) \delta(\theta)+\left(\tau_{0}+\mu\right)\left(\begin{array}{cc}
b_{11} & 0 \\
b_{21} & b_{22}
\end{array}\right) \delta(\theta+1)
$$

where $\delta$ is the Dirac delta function, then (3.3) is satisfied.

For $\phi \in C\left([-1,0], R^{2}\right)$, we define

$$
\begin{gathered}
A(\mu) \phi= \begin{cases}\frac{d \phi(\theta)}{d \theta}, & -1 \leq \theta<0, \\
\int_{-1}^{0} d \eta(\theta, \mu) \phi(\theta), & \theta=0\end{cases} \\
R(\mu) \phi= \begin{cases}0, & -1 \leq \theta<0 \\
F(\mu, \phi), & \theta=0 .\end{cases}
\end{gathered}
$$


Then system (3.1) can be transformed into the following operator equation

$$
\dot{u}(t)=A(\mu) u_{t}+R(\mu) u_{t}
$$

The adjoint operator $A^{*}$ of $A(0)$ is defined by

$$
A^{*}(\varphi)= \begin{cases}-\frac{d \varphi(s)}{d s}, & 0<s \leq 1, \\ \int_{-1}^{0} d \eta^{T}(\xi, 0) \varphi(-\xi), & s=0\end{cases}
$$

associated with a bilinear form

$$
\langle\varphi(s), \phi(\theta)\rangle=\bar{\varphi}^{T}(0) \phi(0)-\int_{\theta=-1}^{0} \int_{\xi=0}^{\theta} \bar{\varphi}^{T}(\xi-\theta) d \eta(\theta) \phi(\xi) d \xi,
$$

where $\eta(\theta)=\eta(\theta, 0)$.

From the above discussion, we know that that $\pm i \omega_{0} \tau_{0}$ are eigenvalues of $A(0)$ and they are also eigenvalues of $A^{*}$.

We assume that $q(\theta)=\left(1, q_{2}\right)^{T} e^{i \tau_{0} \omega_{0} \theta}$ are the eigenvectors of $A(0)$ belonging to the eigenvalue $i \tau_{0} \omega_{0}$ and $q^{*}(\theta)=D\left(1, q_{2}^{*}\right) e^{i \tau_{0} \omega_{0} \theta}$ are the eigenvectors of $A^{*}$ belonging to $-i \tau_{0} \omega_{0}$. Thus,

$$
A(0) q(\theta)=i \tau_{0} \omega_{0} q(\theta), \quad A^{*}(0) q^{*}(\theta)=-i \tau_{0} \omega_{0} q^{*}(\theta) .
$$

Then, we can obtain

$$
\begin{gathered}
q_{2}=\frac{i \omega_{0}-a_{11}-b_{11}}{a_{12}}, \quad q_{2}^{*}=-\frac{i \omega_{0}+a_{11}+b_{11}}{b_{21}} b_{21} e^{i \tau_{0} \omega_{0}}, \\
\bar{D}=\left[1+q_{2} q_{2}^{*}+\left(b_{11}+b_{21} q_{2}^{*}+b_{22} q_{2} q_{2}^{*}\right) e^{i \tau_{0} \omega_{0}}\right]^{-1} .
\end{gathered}
$$

Next, we get the coefficients used in determining the important quantities of the periodic solution by using a computation process similar to that in [15]:

$$
\begin{gathered}
g_{20}=2 \tau_{0} \bar{D}\left\{a_{13}+a_{14} q^{(2)}(0)+a_{15}\left(q^{(2)}(0)\right)^{2}-q^{(1)}(-1)\right. \\
+\bar{q}_{2}^{*}\left(b_{23}\left(q^{(1)}(-1)\right)^{2}+b_{24} q^{(1)}(-1) q^{(2)}(0)\right. \\
\left.\left.+b_{25} q^{(1)}(-1) q^{(2)}(-1)+b_{26} q^{(2)}(0) q^{(2)}(-1)\right)\right\} \\
g_{11}=\tau_{0} \bar{D}\left\{2 a_{13}+a_{14}\left(q^{(2)}(0)+\bar{q}^{(2)}(0)\right)+2 a_{15} q^{(2)}(0) \bar{q}^{(2)}(0)-q^{(1)}(-1)\right. \\
-\bar{q}^{(1)}(-1)+\bar{q}_{2}^{*}\left(2 b_{23} q^{(1)}(-1) \bar{q}^{(1)}(-1)\right.
\end{gathered}
$$


Journal of Applied Mathematics

$$
\begin{aligned}
& +b_{24}\left(q^{(1)}(-1) \bar{q}^{(2)}(0)+\bar{q}^{(1)}(-1) q^{(2)}(0)\right) \\
& +b_{25}\left(q^{(1)}(-1) \bar{q}^{(2)}(-1)+\bar{q}^{(1)}(-1) q^{(2)}(-1)\right) \\
& \left.\left.+b_{26}\left(q^{(2)}(0) \bar{q}^{(2)}(-1)+\bar{q}^{(2)}(0) q^{(2)}(-1)\right)\right)\right\}, \\
& g_{02}=2 \tau_{0} \bar{D}\left\{a_{13}+a_{14} \bar{q}^{(2)}(0)+a_{15}\left(\bar{q}^{(2)}(0)\right)^{2}-\bar{q}^{(1)}(-1)\right. \\
& +\bar{q}_{2}^{*}\left(b_{23}\left(\bar{q}^{(1)}(-1)\right)^{2}+b_{24} \bar{q}^{(1)}(-1) \bar{q}^{(2)}(0)\right. \\
& \left.\left.+b_{25} \bar{q}^{(1)}(-1) \bar{q}^{(2)}(-1)+b_{26} \bar{q}^{(2)}(0) \bar{q}^{(2)}(-1)\right)\right\}, \\
& g_{21}=2 \tau_{0} \bar{D}\left\{a_{13}\left(W_{20}^{(1)}(0)+2 W_{11}^{(1)}(0)\right)\right. \\
& +a_{14}\left(\frac{1}{2} W_{20}^{(2)}(0)+W_{11}^{(2)}(0)+\frac{1}{2} W_{20}^{(1)}(0) \bar{q}^{(2)}(0)+W_{11}^{(1)}(0) q^{(2)}(0)\right) \\
& +a_{15}\left(W_{20}^{(2)}(0) \bar{q}^{(2)}(0)+2 W_{11}^{(2)}(0) q^{(2)}(0)\right) \\
& -\left(W_{11}^{(1)}(-1)+\frac{1}{2} W_{20}^{(1)}(-1)+W_{11}^{(1)}(0) q^{(1)}(-1)+\frac{1}{2} W_{20}^{(1)}(0) \bar{q}^{(1)}(-1)\right)+3 a_{16} \\
& +a_{17}\left(2 q^{(2)}(0)+\bar{q}^{(2)}(0)\right)+a_{18}\left(2 q^{(2)}(0) \bar{q}^{(2)}(0)+\left(q^{(2)}(0)\right)^{2}\right) \\
& +3 a_{19}\left(q^{(2)}(0)\right)^{2} \bar{q}^{(2)}(0) \\
& +\bar{q}_{2}^{*}\left(b_{23}\left(W_{20}^{(1)}(-1) \bar{q}^{(1)}(-1)+2 W_{11}^{(1)}(-1) q^{(1)}(-1)\right)\right. \\
& +b_{24}\left(\frac{1}{2} W_{20}^{(2)}(0) \bar{q}^{(1)}(-1)+W_{11}^{(2)}(0) q^{(1)}(-1)\right. \\
& \left.+\frac{1}{2} W_{20}^{(1)}(-1) \bar{q}^{(2)}(0)+W_{11}^{(1)}(-1) q^{(2)}(0)\right) \\
& +b_{25}\left(\frac{1}{2} W_{20}^{(1)}(-1) \bar{q}^{(2)}(-1)+W_{11}^{(1)}(-1) q^{(2)}(-1)\right. \\
& \left.+\frac{1}{2} W_{20}^{(2)}(-1) \bar{q}^{(1)}(-1)+W_{11}^{(2)}(-1) q^{(1)}(-1)\right) \\
& +b_{26}\left(\frac{1}{2} W_{20}^{(2)}(0) \bar{q}^{(2)}(-1)+W_{11}^{(2)}(0) q^{(2)}(-1)\right. \\
& \left.+\frac{1}{2} W_{20}^{(2)}(-1) \bar{q}^{(2)}(0)+W_{11}^{(2)}(-1) q^{(2)}(0)\right) \\
& +3 b_{27}\left(q^{(1)}(-1)\right)^{2} \bar{q}^{(1)}(-1)
\end{aligned}
$$




$$
\begin{aligned}
& +b_{28}\left(\left(q^{(1)}(-1)\right)^{2} \bar{q}^{(2)}(0)+2 q^{(1)}(-1) \bar{q}^{(1)}(-1) q^{(2)}(0)\right) \\
& \left.\left.+b_{29}\left(\left(q^{(1)}(-1)\right)^{2} \bar{q}^{(2)}(-1)+2 q^{(1)}(-1) \bar{q}^{(1)}(-1) q^{(2)}(-1)\right)\right)\right\}
\end{aligned}
$$

with

$$
\begin{gathered}
W_{20}(\theta)=\frac{i g_{20} q(0)}{\tau_{0} \omega_{0}} e^{i \tau_{0} \omega_{0} \theta}+\frac{i \bar{g}_{02} \bar{q}(0)}{3 \tau_{0} \omega_{0}} e^{-i \tau_{0} \omega_{0} \theta}+E_{1} e^{2 i \tau_{0} \omega_{0} \theta}, \\
W_{11}(\theta)=-\frac{i g_{11} q(0)}{\tau_{0} \omega_{0}} e^{i \tau_{0} \omega_{0} \theta}+\frac{i \bar{g}_{11} \bar{q}(0)}{\tau_{0} \omega_{0}} e^{-i \tau_{0} \omega_{0} \theta}+E_{2},
\end{gathered}
$$

where $E_{1}$ and $E_{2}$ can be computed as the following equations, respectively

$$
\begin{gathered}
E_{1}=2\left(\begin{array}{l}
M_{11} \\
M_{21}
\end{array}\right) \times\left(\begin{array}{cc}
2 i \omega_{0}-a_{11}-b_{11} & -a_{12} \\
-c_{21} e^{-2 i \tau_{0} \omega_{0}} & 2 i \omega_{0}-c_{22} e^{-2 i \tau_{0} \omega_{0}}
\end{array}\right)^{-1}, \\
E_{2}=-\left(\begin{array}{c}
N_{11} \\
N_{21}
\end{array}\right) \times\left(\begin{array}{cc}
a_{11}+b_{11} & a_{12} \\
c_{21} & c_{22}
\end{array}\right)^{-1}
\end{gathered}
$$

with

$$
\begin{aligned}
M_{11}= & a_{13}+a_{14} q^{(2)}(0)+a_{15}\left(q^{(2)}(0)\right)^{2}-q^{(1)}(-1), \\
M_{21}= & b_{23}\left(q^{(1)}(-1)\right)^{2}+b_{24} q^{(1)}(-1) q^{(2)}(0)+b_{25} q^{(1)}(-1) q^{(2)}(-1)+b_{26} q^{(2)}(0) q^{(2)}(-1), \\
N_{11}= & 2 a_{13}+a_{14}\left(q^{(2)}(0)+\bar{q}^{(2)}(0)\right)+2 a_{15} q^{(2)}(0) \bar{q}^{(2)}(0)-q^{(1)}(-1)-\bar{q}^{(1)}(-1), \\
N_{21}= & 2 b_{23} q^{(1)}(-1) \bar{q}^{(1)}(-1)+b_{24}\left(q^{(1)}(-1) \bar{q}^{(2)}(0)+\bar{q}^{(1)}(-1) q^{(2)}(0)\right) \\
& +b_{25}\left(q^{(1)}(-1) \bar{q}^{(2)}(-1)+\bar{q}^{(1)}(-1) q^{(2)}(-1)\right) \\
& +b_{26}\left(q^{(2)}(0) \bar{q}^{(2)}(-1)+\bar{q}^{(2)}(0) q^{(2)}(-1)\right) .
\end{aligned}
$$

Therefore, we can calculate the following values:

$$
\begin{gathered}
C_{1}(0)=\frac{i}{2 \tau_{0} \omega_{0}}\left(g_{11} g_{20}-2\left|g_{11}\right|^{2}-\frac{\left|g_{02}\right|^{2}}{3}\right)+\frac{g_{21}}{2}, \\
\mu_{2}=-\frac{\operatorname{Re}\left\{C_{1}(0)\right\}}{\operatorname{Re}\left\{\lambda^{\prime}\left(\tau_{0}\right)\right\}}, \\
\beta_{2}=2 \operatorname{Re}\left\{C_{1}(0)\right\} .
\end{gathered}
$$

Based on the discussion above, we can obtain the following results. 

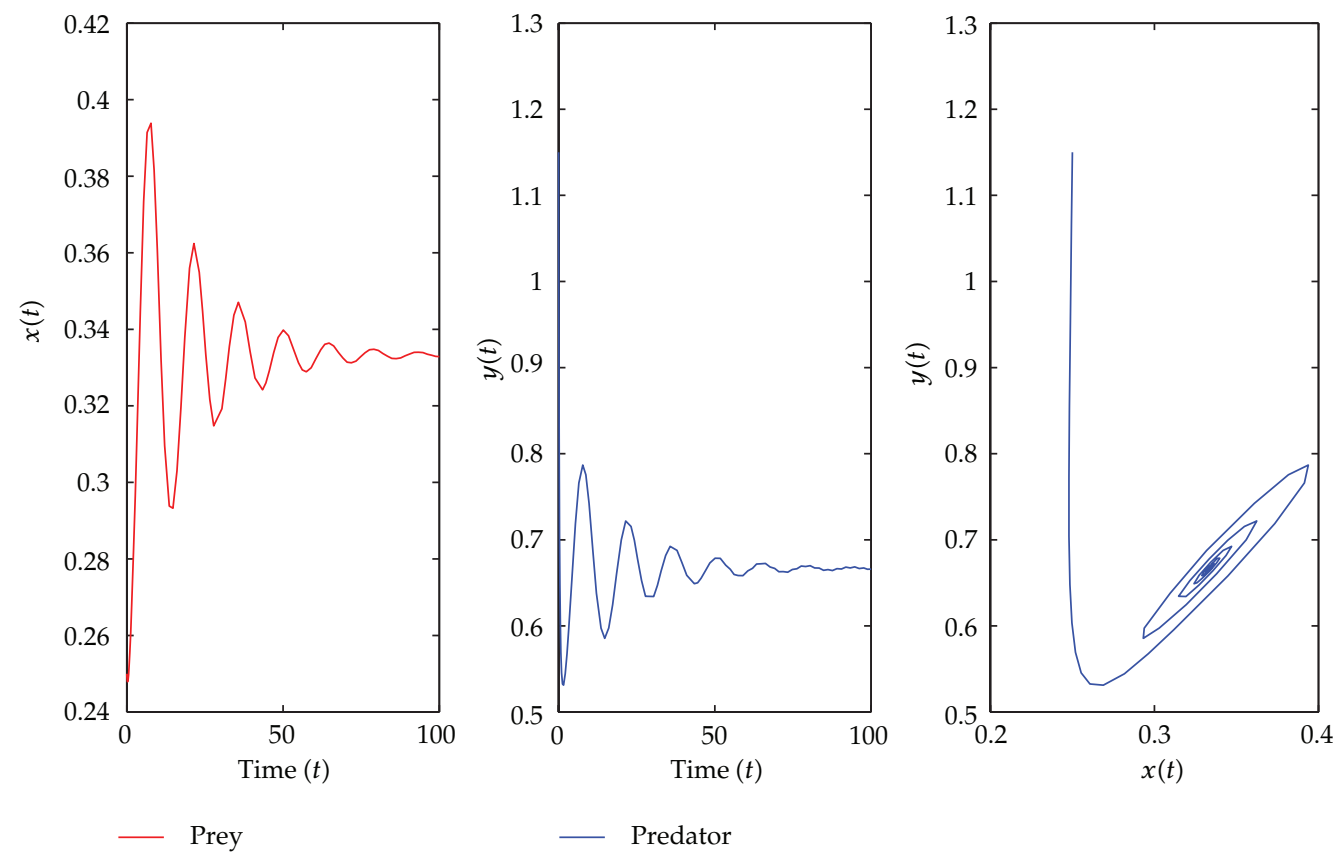

Figure 1: $E(0.3333,0.6666)$ is asymptotically stable when $\tau_{1}=3.15<\tau_{10}=3.7457$ with initial value 0.25 , 1.15 .

Theorem 3.1. The direction of the Hopf bifurcation is determined by the sign of $\mu_{2}$ : if $\mu_{2}>0\left(\mu_{2}<0\right)$, then the Hopf bifurcation is supercritical (subcritical). The stability of bifurcating periodic solutions is determined by the sign of $\beta_{2}$ : if $\beta_{2}<0\left(\beta_{2}>0\right)$, the bifurcating periodic solutions are stable (unstable).

\section{Numerical Example}

In this section, to illustrate the analytical results obtained in the previous sections, we present some numerical simulations. Let $\alpha=1, \beta=1, \delta=2$, then we have the following particular case of system (1.4):

$$
\begin{gathered}
\frac{d x}{d t}=x(t)\left(1-x\left(t-\tau_{1}\right)\right)-\frac{x(t) y(t)}{x(t)+y(t)} \\
\frac{d y}{d t}=y(t)\left(2-\frac{y\left(t-\tau_{2}\right)}{x\left(t-\tau_{2}\right)}\right) .
\end{gathered}
$$

Obviously, $1+\alpha \delta=3>\delta=2$. Thus, the condition $\left(H_{1}\right)$ holds. Then we can get the unique positive equilibrium $E(0.3333,0.6666)$ of system (4.1). By a simple computation, $A+B+C=$ $2.1110>0, D+E=0.5998>0$. Namely, the condition $\left(H_{2}\right)$ holds. 

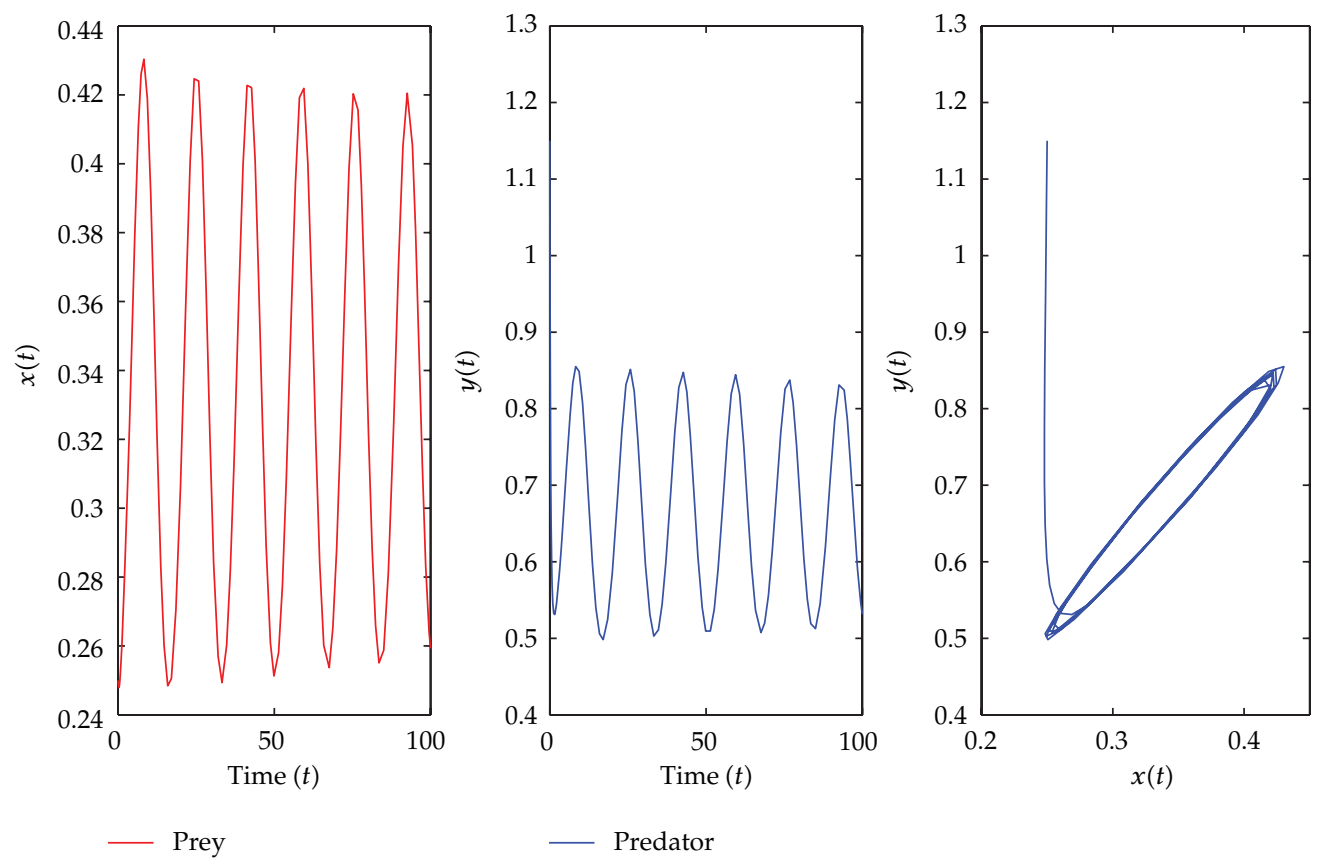

Figure 2: $E(0.3333,0.6666)$ is unstable when $\tau_{1}=4.05>\tau_{10}=3.7457$ with initial value $0.25,1.15$.
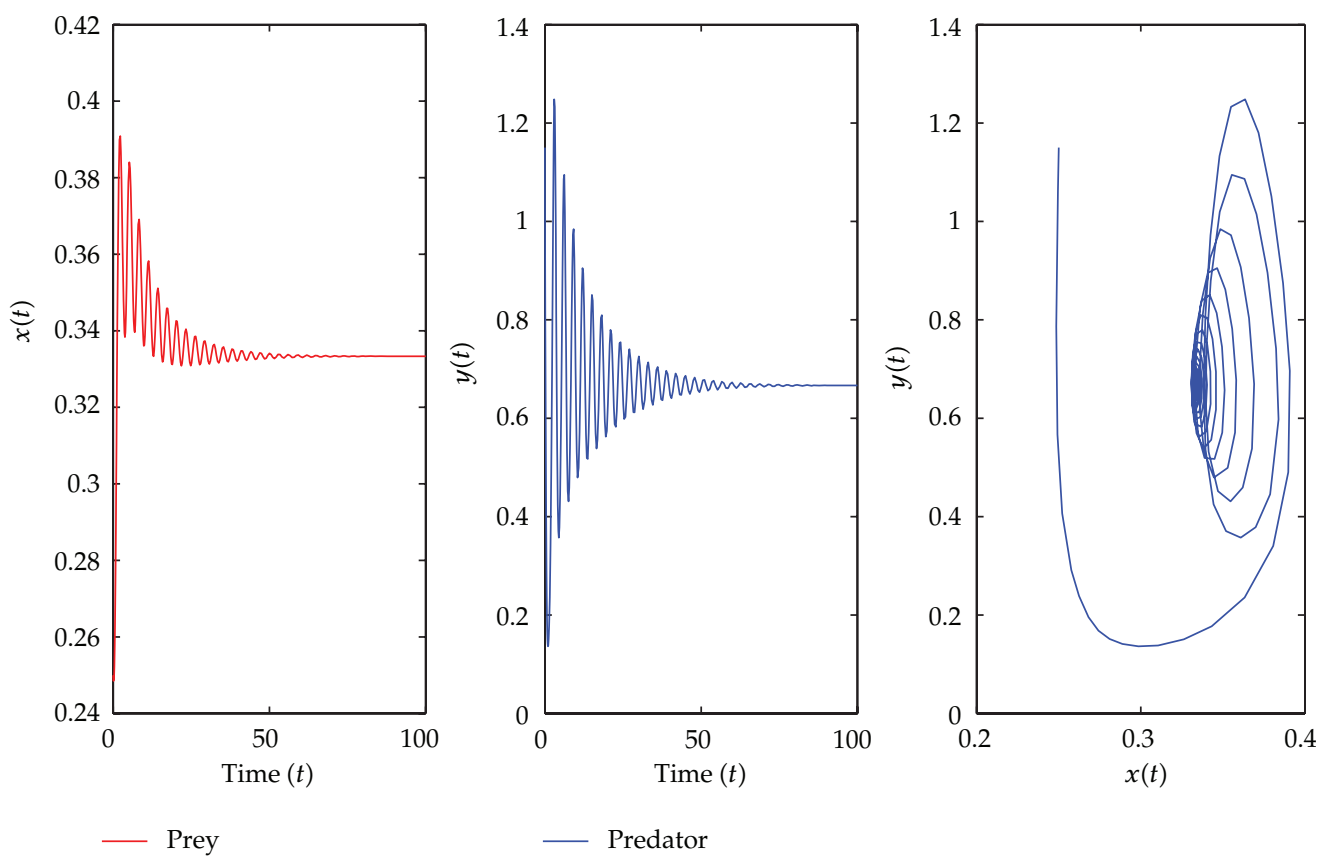

Figure 3: $E(0.3333,0.6666)$ is asymptotically stable when $\tau_{2}=0.68<\tau_{20}=0.7311$ with initial value 0.25 , 1.15 . 

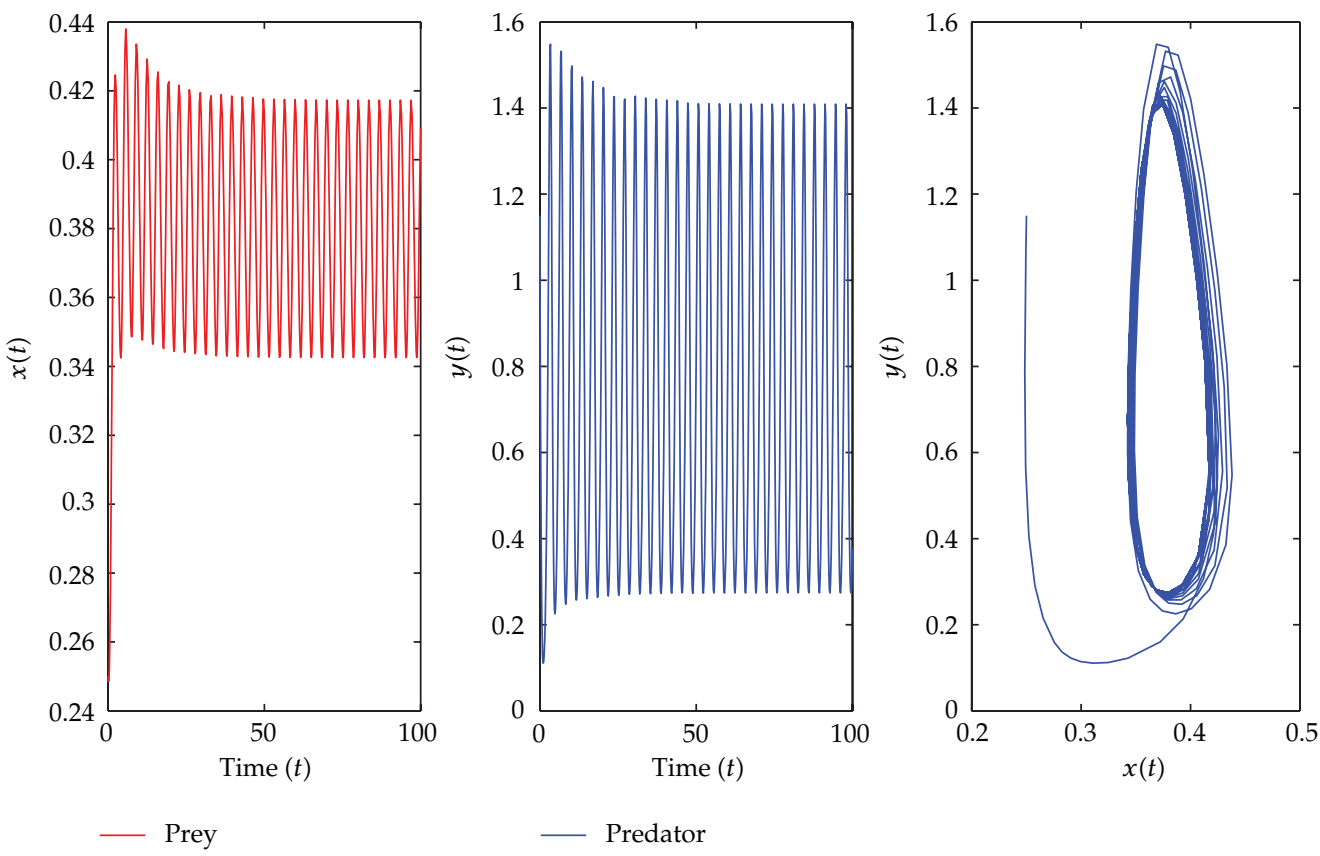

Figure 4: $E(0.3333,0.6666)$ is unstable when $\tau_{2}=0.75>\tau_{10}=0.7311$ with initial value $0.25,1.15$.

Firstly, we can obtain that $D^{2}-E^{2}=-0.4444<0$. Namely, the condition $\left(H_{3}\right)$ is satisfied for $\tau_{1}>0, \tau_{2}=0$. Further, we have $\omega_{10}=0.3734, \tau_{10}=3.7457$. By Theorem 2.1, we can know that the positive equilibrium $E(0.3333,0.6666)$ is asymptotically stable for $\tau_{1} \in\left[0, \tau_{10}\right)$ and unstable when $\tau_{1}>\tau_{10}$. Let $\tau_{1}=3.15 \in\left[0, \tau_{10}\right)$, then the positive equilibrium $E(0.3333,0.6666)$ is asymptotically stable, which can be seen from Figure 1 . When $\tau_{1}=4.05>\tau_{10}=3.7457$, it can be seen from Figure 2 that the positive equilibrium $E(0.3333,0.6666)$ is unstable and a Hopf bifurcation occurs. Similarly, we have $\omega_{20}=2.0189, \tau_{20}=0.7311$. For $\tau_{2}=0.68 \in\left[0, \tau_{20}\right)$, the positive equilibrium $E(0.3333,0.6666)$ is asymptotically stable from Theorem 2.2 and this property can be shown in Figure 3. If $\tau_{2}=0.75>\tau_{20}=0.7311$, the positive equilibrium $E(0.3333,0.6666)$ is unstable and a Hopf bifurcation occurs, and the corresponding waveform and phase plots are shown in Figure 4.

Secondly, we can get $\omega_{0}=2.2937, \tau_{0}=0.7015$, and $\lambda^{\prime}\left(\tau_{0}\right)=0.9911+1.7808 i$ for $\tau_{1}=$ $\tau_{2}=\tau>0$. From Theorem 2.3 , we know that $E(0.3333,0.6666)$ is asymptotically stable for $\tau \in\left[0, \tau_{0}\right)$, which can be illustrated by Figure 5 . As can be seen from Figure 5 that when $\tau=0.64 \in\left[0, \tau_{0}\right)$ the positive equilibrium $E(0.3333,0.6666)$ is asymptotically stable. However, if $\tau=0.74>\tau_{0}=0.7015$, then the positive equilibrium $E(0.3333,0.6666)$ becomes unstable and a family of bifurcated periodic solutions occur, which is illustrated by Figure 6 . In addition, from (3.18), we get $\mu_{2}=139.3>0, \beta_{2}=-276.12<0$. Thus, by Theorem 3.1, we know that the Hopf bifurcation is supercritical and the bifurcated periodic solutions are stable.

Lastly, regard $\tau_{1}$ as a parameter and let $\tau_{2}=0.5 \in\left(0, \tau_{20}\right)$, we can obtain that $\omega_{*}=$ 0.3758. Further we have $\tau_{1 *}=3.7688$. Let $\tau_{1}=3.25 \in\left[0, \tau_{1 *}\right)$, we can know that the positive equilibrium $E(0.3333,0.6666)$ is asymptotically stable from Theorem 2.4 , which can be shown by Figure 7 . When $\tau_{1}=4.15>\tau_{1 *}=3.7688$ then the positive equilibrium $E(0.3333,0.6666)$ becomes unstable and a Hopf bifurcation occurs, which can be illustrated in Figure 8 . 

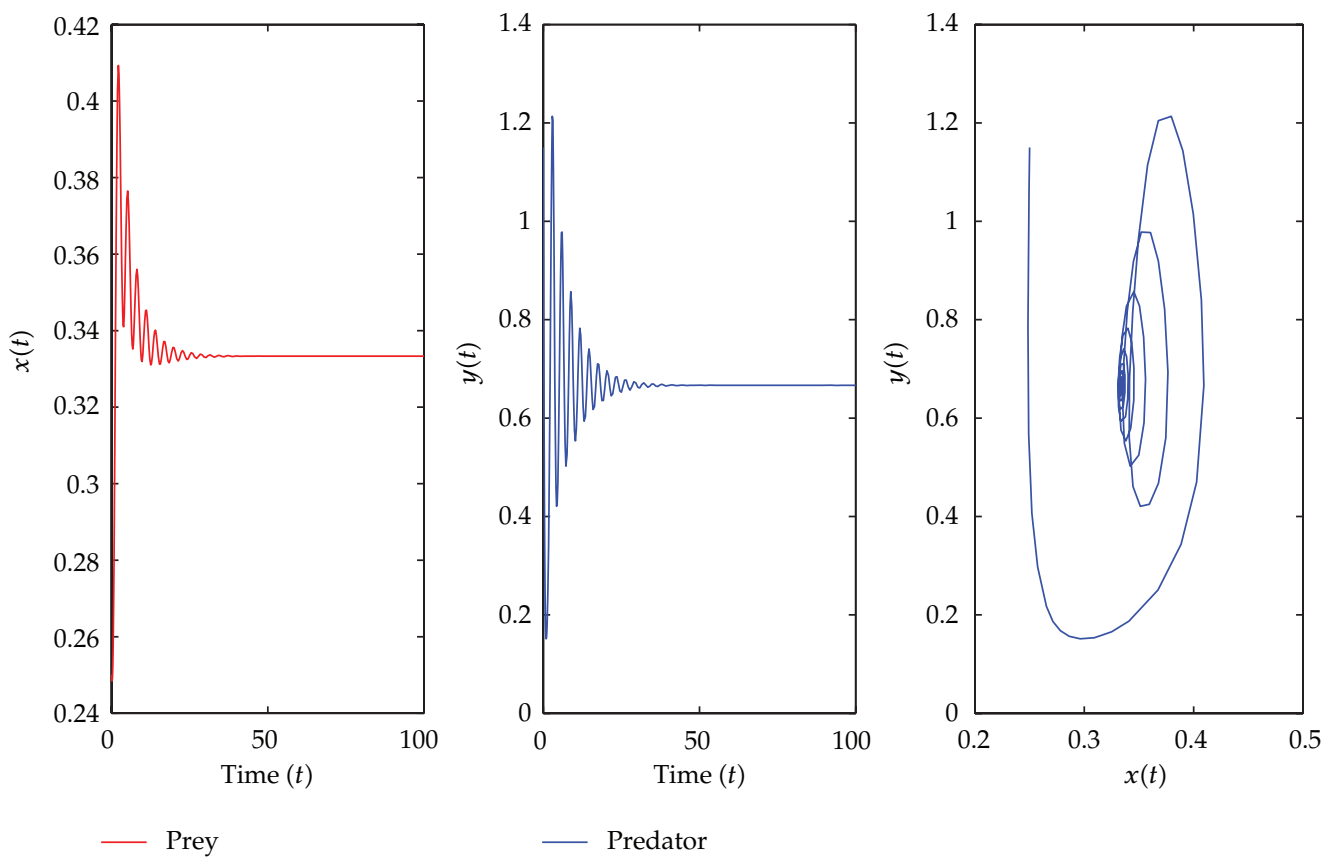

Figure 5: $E(0.3333,0.6666)$ is asymptotically stable when $\tau=0.64<\tau_{0}=0.7015$ with initial value $0.25,1.15$.
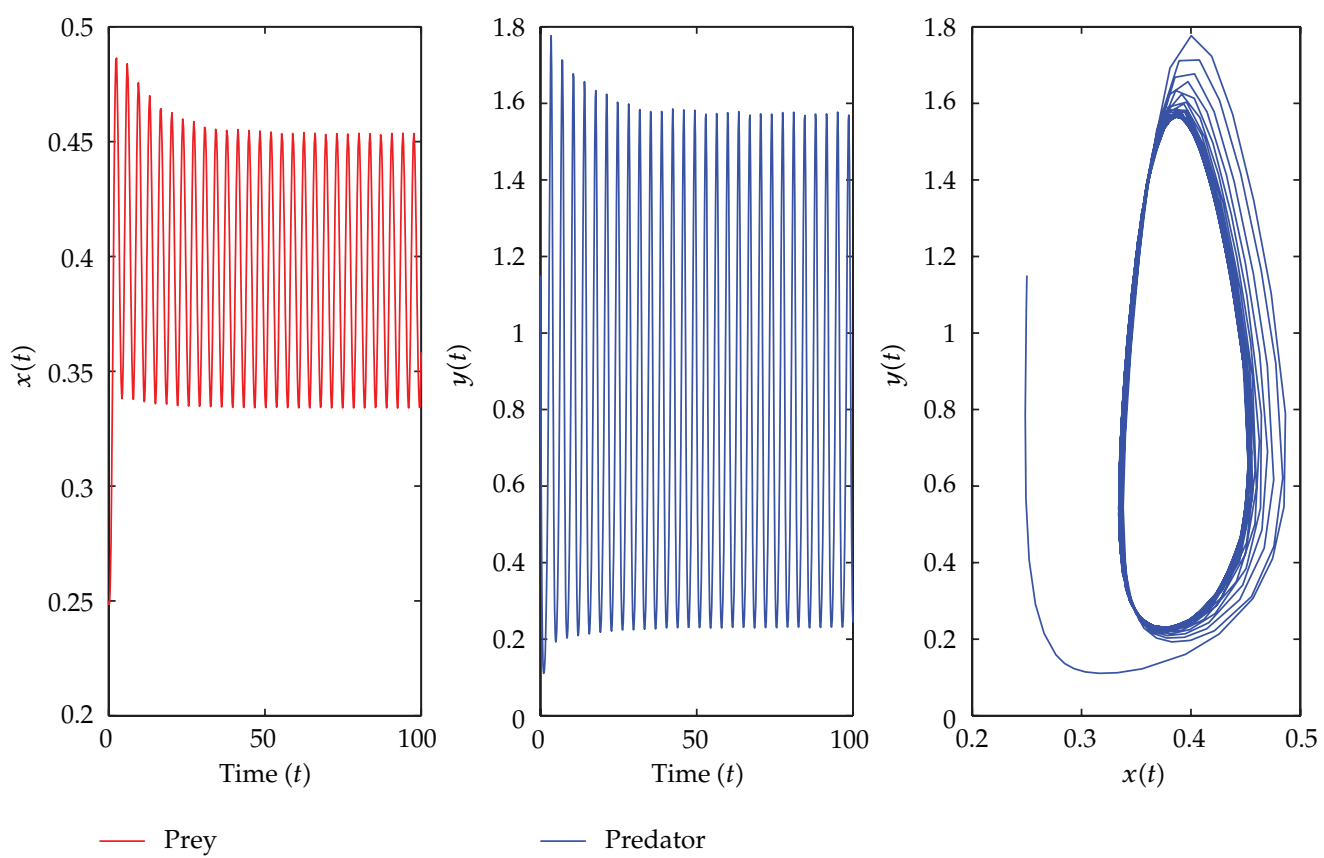

— Predator

Figure 6: $E(0.3333,0.6666)$ is unstable when $\tau=0.74>\tau_{0}=0.7015$ with initial value $0.25,1.15$. 
Journal of Applied Mathematics
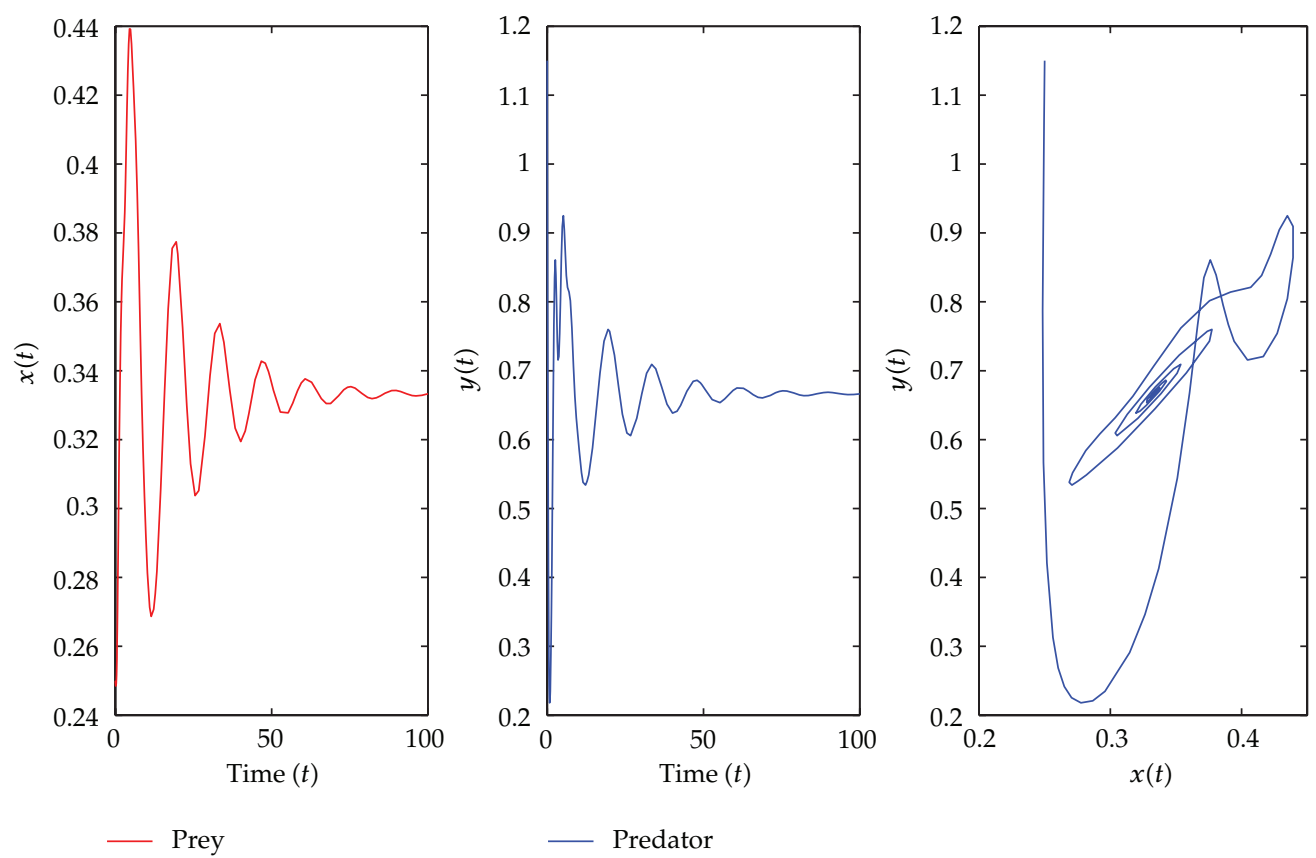

Figure 7: $E(0.3333,0.6666)$ is asymptotically stable when $\tau_{1}=3.25<\tau_{1 *}=3.7457$ with $\tau_{2}=0.5$ and initial value $0.25,1.15$.
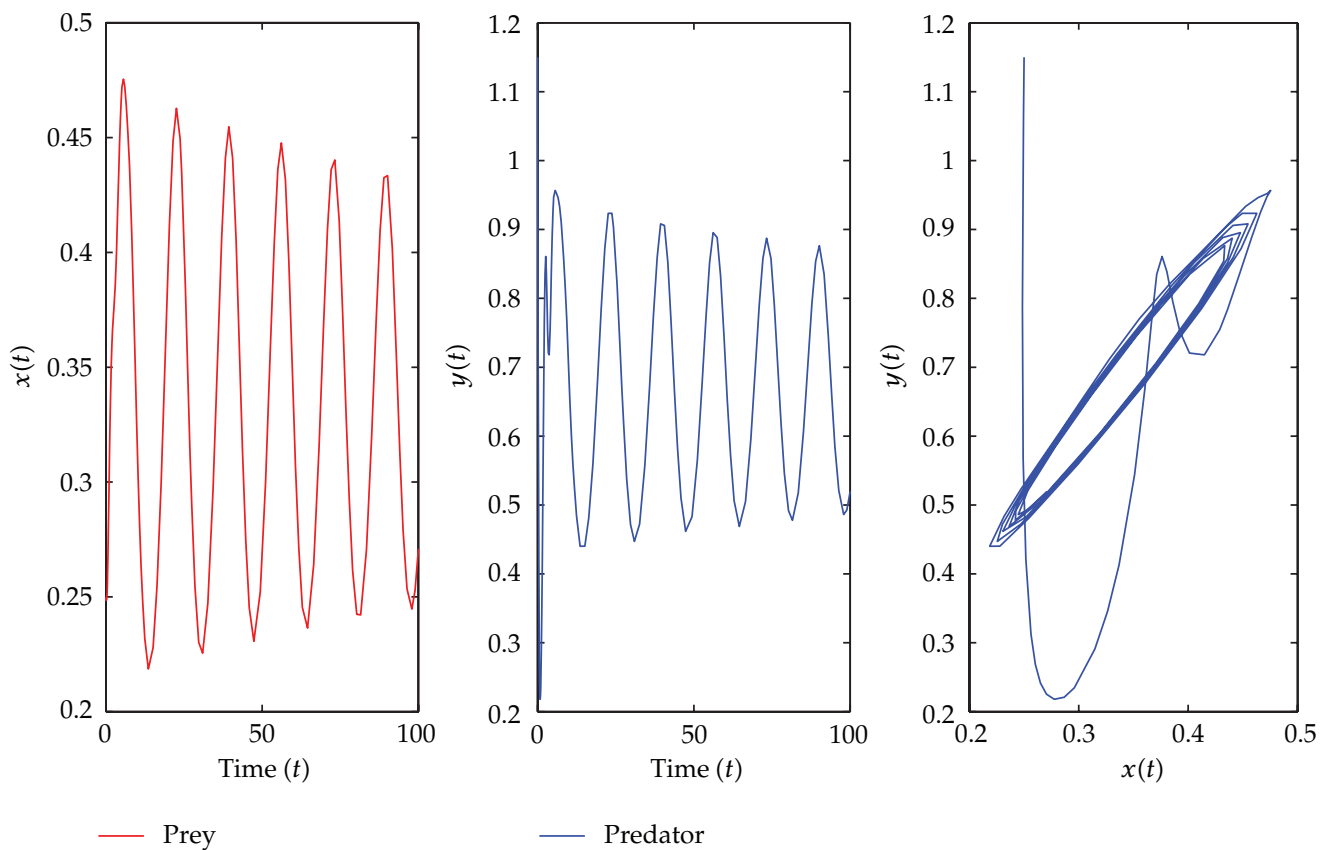

Figure 8: $E(0.3333,0.6666)$ is unstable when $\tau_{1}=4.15>\tau_{1 *}=3.7457$ with with $\tau_{2}=0.5$ and initial value $0.25,1.15$. 


\section{Conclusion}

In the present paper, a Holling-Tanner predator-prey system with ratio-dependent functional response and two delays is investigated. We prove that the system is asymptotically stable under certain conditions. Compared with the system considered in [7], we not only consider the feedback delay of the prey but also the feedback delay of the predator. By choosing the delay as a bifurcation parameter, we show that the Hopf bifurcations can occur as the delay crosses some critical values. Furthermore, we get that the two species could also coexist with some available delays of the prey and the predator. This is valuable from the view of biology. In addition, Saha and Chakrabarti [7] only considered the stability of the system. It is well known that there are also some other behaviors for dynamical systems. Based on this consideration, we investigate the Hopf bifurcation and properties of the bifurcated periodic solutions of the system. The direction and the stability of the bifurcated periodic solutions are determined by applying the normal theory and the center manifold theorem. If the bifurcated periodic solutions are stable, then the two species may coexist in an oscillatory mode from the viewpoint of biology. Some numerical simulations supporting the theoretical results are also included.

\section{Acknowledgments}

The authors are grateful to the referees and the editor for their valuable comments and suggestions on the paper. This work is supported by Anhui Provincial Natural Science Foundation under Grant no. 1208085QA11.

\section{References}

[1] A. A. Berryman, “The origins and evolution of predator-prey theory,” Ecology, vol. 73, no. 5, pp. 1530$1535,1992$.

[2] C. Cosner, D. L. Deangelis, J. S. Ault, and D. B. Olson, "Effects of spatial grouping on the functional response of predators," Theoretical Population Biology, vol. 56, no. 1, pp. 65-75, 1999.

[3] S. B. Hsu, T. W. Hwang, and Y. Kuang, "Rich dynamics of a ratio-dependent one-prey two-predators model," Journal of Mathematical Biology, vol. 43, no. 5, pp. 377-396, 2001.

[4] Y. Kuang, "Rich dynamics of Gause-type ratio-dependent predator-prey system," Fields Institute Communications, vol. 21, pp. 325-337, 1999.

[5] M. Haque and B. L. Li, "A ratio-dependent predator-prey model with logistic growth for the predator population," in Proceedings of 10th International Conference on Computer Modeling and Simulation, pp. 210-215, University of Cambridge, Cambridge, UK, April 2008.

[6] Z. Liang and H. Pan, "Qualitative analysis of a ratio-dependent Holling-Tanner model," Journal of Mathematical Analysis and Applications, vol. 334, no. 2, pp. 954-964, 2007.

[7] T. Saha and C. Chakrabarti, "Dynamical analysis of a delayed ratio-dependent Holling-Tanner predator-prey model," Journal of Mathematical Analysis and Applications, vol. 358, no. 2, pp. 389-402, 2009.

[8] X.-Y. Meng, H.-F. Huo, and H. Xiang, "Hopf bifurcation in a three-species system with delays," Journal of Applied Mathematics and Computing, vol. 35, no. 1-2, pp. 635-661, 2011.

[9] Y.-H. Fan and L.-L. Wang, "Periodic solutions in a delayed predator-prey model with nonmonotonic functional response," Nonlinear Analysis. Real World Applications, vol. 10, no. 5, pp. 3275-3284, 2009.

[10] L. Zhang and C. Lu, "Periodic solutions for a semi-ratio-dependent predator-prey system with Holling IV functional response," Journal of Applied Mathematics and Computing, vol. 32, no. 2, pp. 465477, 2010.

[11] S. Yuan and Y. Song, "Stability and Hopf bifurcations in a delayed Leslie-Gower predator-prey system," Journal of Mathematical Analysis and Applications, vol. 355, no. 1, pp. 82-100, 2009. 
[12] G.-P. Hu and X.-L. Li, "Stability and Hopf bifurcation for a delayed predator-prey model with disease in the prey," Chaos, Solitons \& Fractals, vol. 45, no. 3, pp. 229-237, 2012.

[13] F. Lian and Y. Xu, "Hopf bifurcation analysis of a predator-prey system with Holling type IV functional response and time delay," Applied Mathematics and Computation, vol. 215, no. 4, pp. 14841495, 2009.

[14] B. D. Hassard, N. D. Kazarinoff, and Y. H. Wan, Theory and Applications of Hopf Bifurcation, Cambridge University Press, Cambridge, UK, 1981.

[15] T. K. Kar and A. Ghorai, "Dynamic behaviour of a delayed predator-prey model with harvesting," Applied Mathematics and Computation, vol. 217, no. 22, pp. 9085-9104, 2011. 


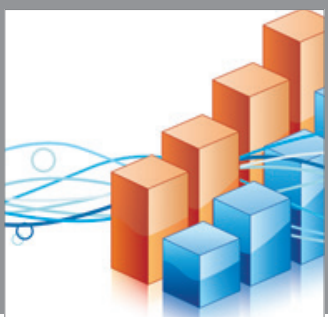

Advances in

Operations Research

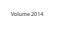

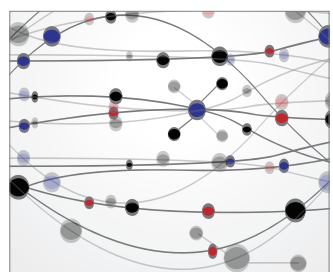

\section{The Scientific} World Journal
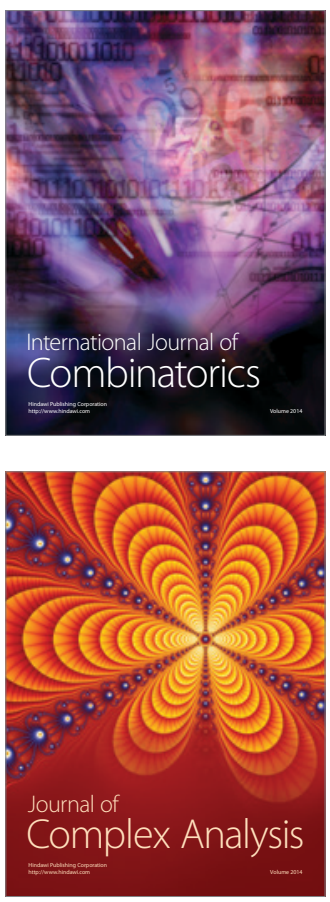

International Journal of

Mathematics and

Mathematical

Sciences
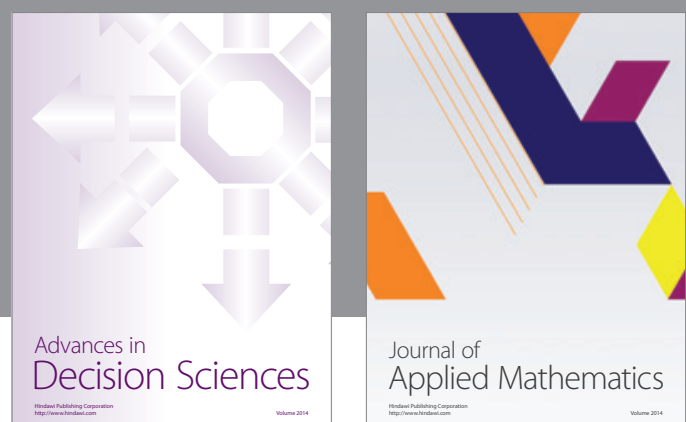

Journal of

Applied Mathematics
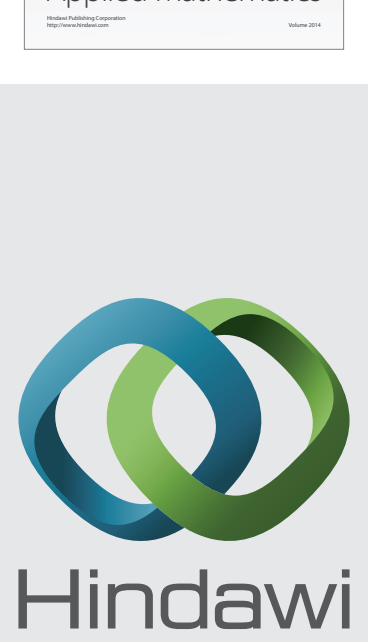

Submit your manuscripts at http://www.hindawi.com
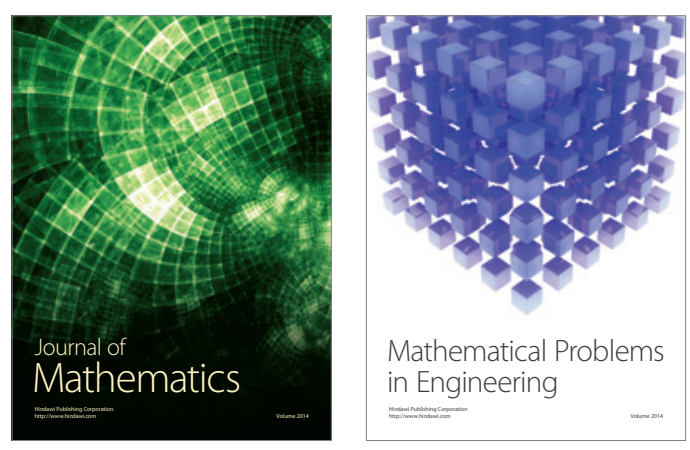

Mathematical Problems in Engineering
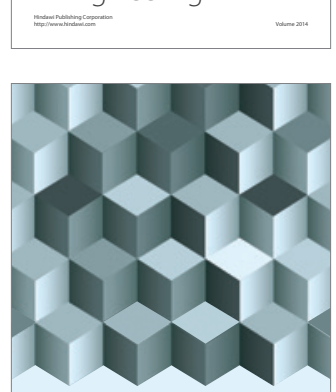

Journal of

Function Spaces
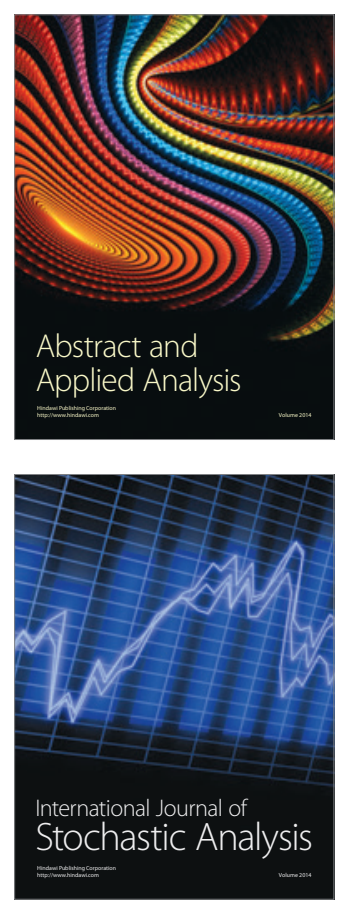

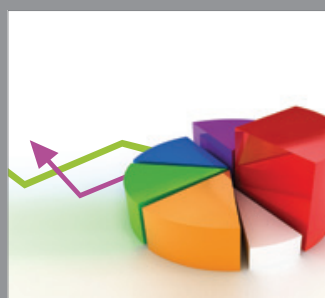

ournal of

Probability and Statistics

Promensencen
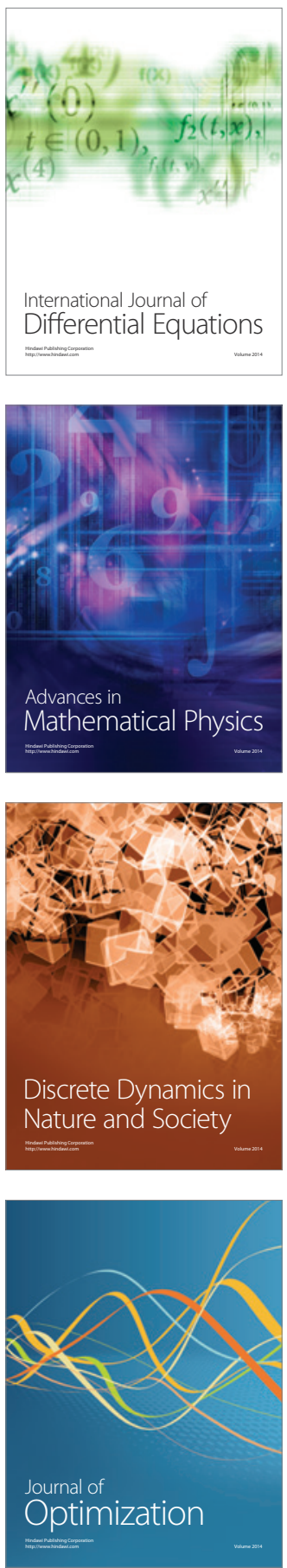